[Vicino Oriente XXI (2017), pp. 265-271]

\title{
BOLLETTINO DELLE ATTIVITÀ DEL MUSEO DEL VICINO ORIENTE, EGITTO E MEDITERRANEO DELLA SAPIENZA, ANNO 2017
}

\author{
Daria Montanari - Sapienza Università di Roma
}

\begin{abstract}
Museum VOEM organized during 2017 exhibits, activities, workshops, conferences and seminars, for the wide public, for scholars and researchers. MVOEM has been monitoring collections and has promoted numerous research projects, studies and analyses on items and objects kept.
\end{abstract}

Keywords: museum management; cultural policy; research; exhibition; training activities

Durante il terzo anno di vita il Museo del Vicino Oriente, Egitto e Mediterraneo ha promosso una serie di attività dedicate ad un ampio pubblico (fig. 1), bambini, studenti, amatori e studiosi, ed ha partecipato alle numerose iniziative organizzate dal Polo Museale Sapienza.

La conservazione e il monitoraggio delle collezioni sono stati portati avanti parallelamente ad alcuni progetti di ricerca (§§ 4.-5.).

Contemporaneamente si è svolta l'ordinaria attività di ricerca sulle collezioni che lo costituiscono, con la partecipazione di studiosi, studenti, tesisti, specializzandi e dottorandi.

Le tre missioni, la divulgazione (§ 1.), la formazione (§ 2.) e la ricerca (§ 3.), sono state perseguite con la partecipazione dello staff del Museo, composto da studenti, laureandi, studenti della Scuola di Specializzazione in Beni Archeologici e della Scuola di Dottorato in Archeologia.

\section{DiVULGAZIONE E OUTREACH}

Nel corso del 2017 una serie di conferenze, seminari, visite guidate ed eventi sono stati organizzati, per un pubblico amatoriale ed esperto, per le scuole inferiori e superiori, nonché per studenti universitari e studiosi.

Numerosi laboratori didattici (fig. 2), incentrati su alcuni temi fondamentali nelle culture del Vicino Oriente e dell'Egitto antichi, quali le prime scritture e l'alfabeto, l'agricoltura e la navigazione, sono stati organizzati con forme interattive di apprendimento ludico.

Per gli studenti delle scuole elementari sono state effettuate nel corso del primo semestre dell'anno 2017 cicli di visite guidate, con percorsi tematici attagliati ai programmi didattici.

Il MVOEM ha aderito nel maggio 2017 all'iniziativa promossa dal Polo Museale Sapienza 'Sabato al Museo', nei giorni di sabato 6, 13, 20 e 27, proponendo un calendario speciale di visite guidate e laboratori didattici, destinato ai bambini tra i 6 e i 12 anni.

Lunedì 8 maggio è stata ricevuta la delegazione cinese THUS Holding.

Sabato 20 maggio, in occasione della "Notte dei Musei” è stata inaugurata l'esposizione intitolata Lost in the Shell. Tale evento è stato dedicato alla presentazione della scoperta di una cachette, da parte della Missione archeologica della Sapienza a Gerico in collaborazione con il Ministero del Turismo e delle Antichità della Palestina. Gli oggetti tesaurizzati nel nascondiglio sono cinque conchiglie nilotiche, queste sono state portate nel MVOEM per lo studio, il restauro e la documentazione. 
In occasione di Porte Aperte alla Sapienza 2017 - XXI Edizione, 11-13 luglio, sono state proposte alcune visite guidate tematiche.

Lunedì 18 settembre è stato accolto il Tour Erasmus Sapienza dedicato a tutti gli studenti stranieri e sabato 23 settembre il Museo ha partecipato con un'apertura straordinaria alla Giornata Europea del Patrimonio - \#GEP 2017. Giovedì 28 settembre il team di studiosi del DNA antico, composto dal Prof. Pierre Zalloua (Harvard University) e dalla Prof.ssa Lisa Matisoo-Smith (University of Otago), sono stati ospitati nel MVOEM per una lectio magistralis.

Sabato 21 ottobre, infine, il MVOEM ha partecipato alla Giornata Internazionale dell'Archeologia, promossa dal Penn Museum di Filadelfia.

\section{FORMAZIONE}

Durante l'anno 2017 sono stati rinnovati i percorsi formativi destinati agli studenti delle scuole superiori, con due progetti Alternanza Scuola Lavoro 2017 (§ 2.1.) e agli studenti universitari, grazie a seminari e conferenze (§ 2.2.), proseguendo le attività inaugurate durante l'anno $2016^{1}$.

\subsection{Alternanza Scuola Lavoro 2017}

Il Museo VOEM ha aderito al progetto Alternanza Scuola Lavoro 2017 promosso dalla Sapienza Università di Roma con la realizzazione di due progetti dal titolo I giovani detectives dell'archeologia. L'indagine archeologica 2.0 [P0025], e Passato e futuro si incontrano: archeologia e geomatica. Tecnologie per comprendere il passato [P0115] (fig. $3)$.

Il primo progetto si inquadra nell'attuale dibattito riguardante le strategie sostenibili per la gestione delle collezioni archeologiche vicino-orientali, ponendo come obbiettivo l'accrescimento della consapevolezza nei giovani nei confronti del tema del traffico illecito di antichità. Questo ha riguardato 36 studenti di 9 licei, Liceo Classico Anco Marzio, Liceo Classico Ugo Foscolo, Liceo Classico Bertrand Russel, Liceo Classico Cornelio Tacito, Liceo Classico Augusto, Liceo Classico Orazio, Liceo Scientifico Stanislao Cannizzaro, Liceo Artistico di Tivoli, e l'Istituto Superiore di Via Sarandì, maturando 40 ore per ciascun studente ed è stato svolto nei mesi di gennaio, febbraio e marzo 2017. Tale progetto è stato articolato in dodici incontri, teorici e applicativi, durante i quali sono stati introdotti i concetti di patrimonio culturale, bene archeologico e salvaguardia, conservazione e musealizzazione, e sono state svolte le ricerche tematiche e compilate le schede di salvaguardia. Gli studenti in tali attività sono stati guidati dal Prof. Lorenzo Nigro come tutor Sapienza e delle Dott.sse Daria Montanari ed Elisabetta Gallo come referenti interni Sapienza.

Il secondo progetto, realizzato in collaborazione con il Dipartimento di Ingegneria Civile, Edile e Ambientale (DICEA) della Sapienza, Prof. Mattia Crespi, si è concentrato sulla realizzazione di modelli tridimensionali metrici dei reperti archeologici conservati nel MVOEM con metodi e tecniche di Geomatica e computer vision, in vista della definizione di un protocollo operativo per la documentazione museale e in funzione di forme di musealizzazione e divulgazione 2.0 e dinamica. Questo ha avuto luogo nei mesi di gennaio,

1 Montanari 2016, 123-124. 
XXI (2017) Bollettino delle attività del Museo del Vicino Oriente, Egitto e Mediterraneo, anno 2017

febbraio e marzo 2017 ed ha riguardato 8 studenti di tre licei, Liceo Scientifico Stanislao Cannizzaro, Istituo Superiore Giueppe Colasanti, Istituto Tecnico Giovanni Giorgi.

\subsection{Seminari universitari}

L'anno 2017 si è aperto con una commemorazione della Professoressa Antonia Ciasca grazie ad una conferenza del ciclo a lei dedicato, tenuta il 10 gennaio dalla Prof.ssa Serena Maria Cecchini (Università di Bologna), “Dal Levante all’Occidente Trasmissione di “modelli” e “disegni” nel Mediterraneo nei primi secoli del I millennio a. C.”.

Il giorno giovedì 16 febbraio ha avuto luogo la Brown Bag Lecture "“Endangered Archaeology' two years on: past and future of the EAMENA project - 'Endangered Archaeology’ due anni dopo: passato e futuro del progetto EAMENA, presentata dal Dott. Andrea Zerbini (University of Oxford).

Mercoledì 12 aprile è stato presentato dal Prof. Lorenzo Nigro il corso COURSERA Alle origini delle civiltà mediterranea: archeologia della città dal levante all'Occidente - III-I millennio a.C.

La Prof.ssa Elisabetta Boaretto (Weizmann Institute of Science) ha tenuto la conferenza La nuova cronologia del Bronzo Antico nel Sud del Levante: integrare metodi di scavo con micro-stratigrafia e radiocarbonio, il giorno martedì 9 maggio.

Mercoledì 10 maggio il Prof. Israel FInkelstein (Tel Aviv University) ha tenuto la Brown Bag Lecture "Megiddo in the Bronze Age. The View from 2017” (fig. 4).

\section{RICERCA}

Gli studi multidisciplinari, analisi di tipo chimico, fisico e archeometrico, su alcuni reperti del Museo, inaugurati nell'anno precedente ${ }^{2}$, sono stati continuati e ampliati. Nuovi progetti di ricerca per l'implementazione di applicativi per la scansione 3D dei reperti sono stati avviati in collaborazione con il Dipartimento di Ingegneria Civile, Edile e Ambientale (DICEA) della Sapienza ${ }^{3}$.

Inoltre, alcuni studi e ricerche sono stati condotti all'interno delle attività didattiche curriculari, per le tesi di Laurea Magistrale, della Scuola di Specializzazione in Beni Archeologici e per i progetti del Dottorato di Ricerca.

\subsection{Lo studio delle prime produzioni ceramiche levantine}

Alcuni frammenti ceramici, provenienti dai siti di Gerico, Khirbet al-Batrawy e Mozia, sono stati sottoposti ad analisi petrografiche, diffrazione a raggi X e scansione al microscopio elettronico, al fine di indagare i processi di produzione e lavorazione. Tale attività di ricerca si inquadra nel progetto di ricerca dedicato all'analisi e alla definizione delle produzioni e degli impasti delle ceramiche nel Levante e nel Mediterraneo ${ }^{4}$.

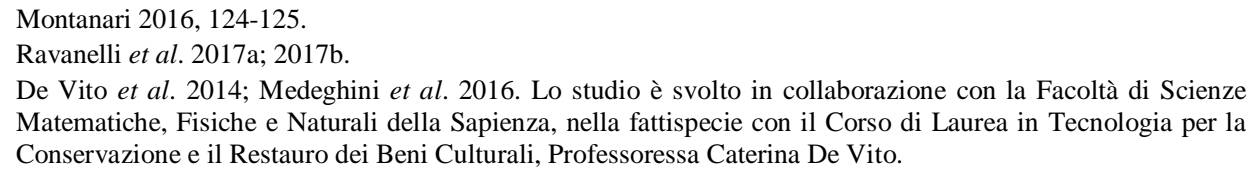

De Vito et al. 2014; Medeghini et al. 2016. Lo studio è svolto in collaborazione con la Facoltà di Scienze Matematiche, Fisiche e Naturali della Sapienza, nella fattispecie con il Corso di Laurea in Tecnologia per la Conservazione e il Restauro dei Beni Culturali, Professoressa Caterina De Vito. 


\subsection{Lo studio dei corredi della necropoli di Achziv}

Nella prima metà dell'anno, è stato effettuato un riesame sistematico dei corredi e delle ceramiche rinvenute nella necropoli di Achziv (vetrina 6) durante gli scavi degli anni 1963 e 1964, condotti dalla Sapienza in collaborazione con Israel Antiquity Authority e diretti da Sabatino Moscati e Moshe Prausnitz ${ }^{5}$.

\subsection{Il pugnale del Bronzo Antico IV da Gerico}

Grazie alla collaborazione con il Centro per le Nanotecnologie Applicate all'Ingegneria (CNIS) della Sapienza, diretto dal Prof. Ruggero Caminiti, è stato possibile sottoporre un pugnale rinvenuto nella necropoli di Gerico esposto nella vetrina 1 del Museo ${ }^{6}$ ai raggi $X$ in scansione di energia (E.D.X.D.) con un diffrattometro non commerciale e all'analisi del Microscopio a Scansione Elettronica (SEM). Tali esami hanno rivelato la composizione metallica dell'arma: rame 93,57\%, stagno 5,87\%, ferro 0,66. Il pugnale da Gerico, dunque, è stato prodotto con una lega di bronzo e costituisce uno dei primi esemplari di tale lega rinvenuti nel sito ${ }^{7}$.

\section{CuRAtela DELLe Collezion}

Nel corso dell'anno 2017 il programma di documentazione impostato nel $2016^{8}$ è stato proseguito. Le collezioni sono state monitorate e salvaguardate, con interventi mirati di pulizia e restauro, e le nuove acquisizioni sono state censite e catalogate (§ 5.).

\section{NUOVE ACCESSIONI}

Sono state registrate nuove accessioni nella Collezione Vicino Oriente del Museo, grazie alla collaborazione con la Rome La Sapienza Expedition to Palestine \& Transjordan (ROSEPAJ), missione archeologica attiva nel 2017 nei siti di Tell es-Sultan/Gerico, e Betlemme $^{9}$, in Palestina, e nel sito di Khirbet al-Batrawy, in Giordania. I reperti acquisiti sono vasellame ceramico, oggetti in pietra e ceramica, e lame di selce, d'età neolitica, del Bronzo Antico e Medio per il sito di Gerico, del Bronzo Antico per il sito di Batrawy.

\section{BIBLIOGRAFIA}

De Vito, C. - Medeghini, L. - Mignardi, S. - Orlandi, D.- Nigro, L. - Spagnoli, F. - Lottici, P.P. BERSANI, D.

2014 Technological fingerprints of Black-GlossWare from Motya (Western Sicily, Italy): Applied Clay Science 88-89 (2014), pp. 202-213.

KAUFMAN, B.

2013 Copper alloys from the 'Enot Shuni cemetery and the origins of bronze metallurgy in the EB IV - MB II Levant: Archaeometry 55 (2013), pp. 663-690.

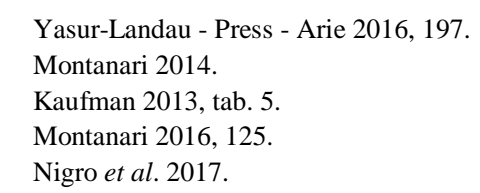


XXI (2017) Bollettino delle attività del Museo del Vicino Oriente, Egitto e Mediterraneo, anno 2017

Medeghini, L. - Fabrizi, L. - De Vito, C. - Mignardi, S. - Nigro, L. - Gallo, E. - Fiaccavento, C.

2016 The ceramic of the "Palace of the Copper Axes" (Khirbet al-Batrawy, Jordan): A palatial special production: Ceramics International 42 (2016), pp. 5952-5962.

MONTANARI, D.

2014 An EB IV dagger from Tell es-Sultan/Jericho: Vicino Oriente XVIII, 2014, pp. 101-111.

2016 Bollettino delle attività del Museo del Vicino Oriente, Egitto e Mediterraneo della Sapienza, anno 2016: Vicino Oriente XX (2016), pp. 121-128.

Nigro, L. - MontAnari, D. - GuARI, A. - TAmbuRrini, M. - Izzo, P. - GhayyadA, M. - Titi, I. -

YASINE, J.

2017 New archaeological features in Bethlehem (Palestine): the Italian-Palestinian rescue season of November 2016: Vicino Oriente XXI (2017), pp. 5-57.

Ravanelli, R. - Nascetti, A. - Di Rita, M. - Nigro, L. - Montanari, D. - Spagnoli, F. - Crespi, M.

2017a 3D Modelling of archaeological small finds by a low-cost range camera: methodology and first results: The International Archives of the Photogrammetry, Remote Sensing and Spatial Information Sciences XLII-5/W1 (2017), pp. 589-592.

Ravanelli, R. - Di Rita, M. - Nascetti, A. - Crespi, M. - Nigro, L. - Montanari, D. - Spagnoli, F.

2017b Penguin 2.0 - Capturing small finds in 3d: Mediterranean Archaeology and Archaeometry 17/2 (2017), pp. 49-56.

Yasur-LANDAu, A. - Press, M.D. - Arie, E.

2016 Rethinking Tel Achziv: An Iron II Architectonic and Ceramic Sequence from Southern Phoenicia: Tel Aviv 43 (2016), pp. 192-224. 


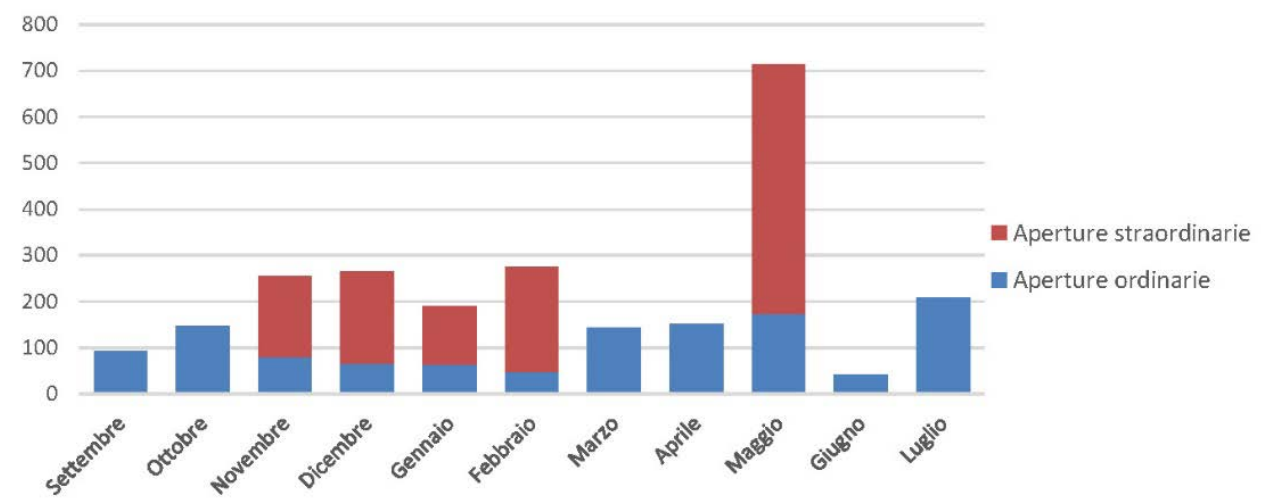

Fig. 1 - Istogramma del numero dei visitatori del Museo VOEM nel periodo Settembre 2016Luglio 2017.

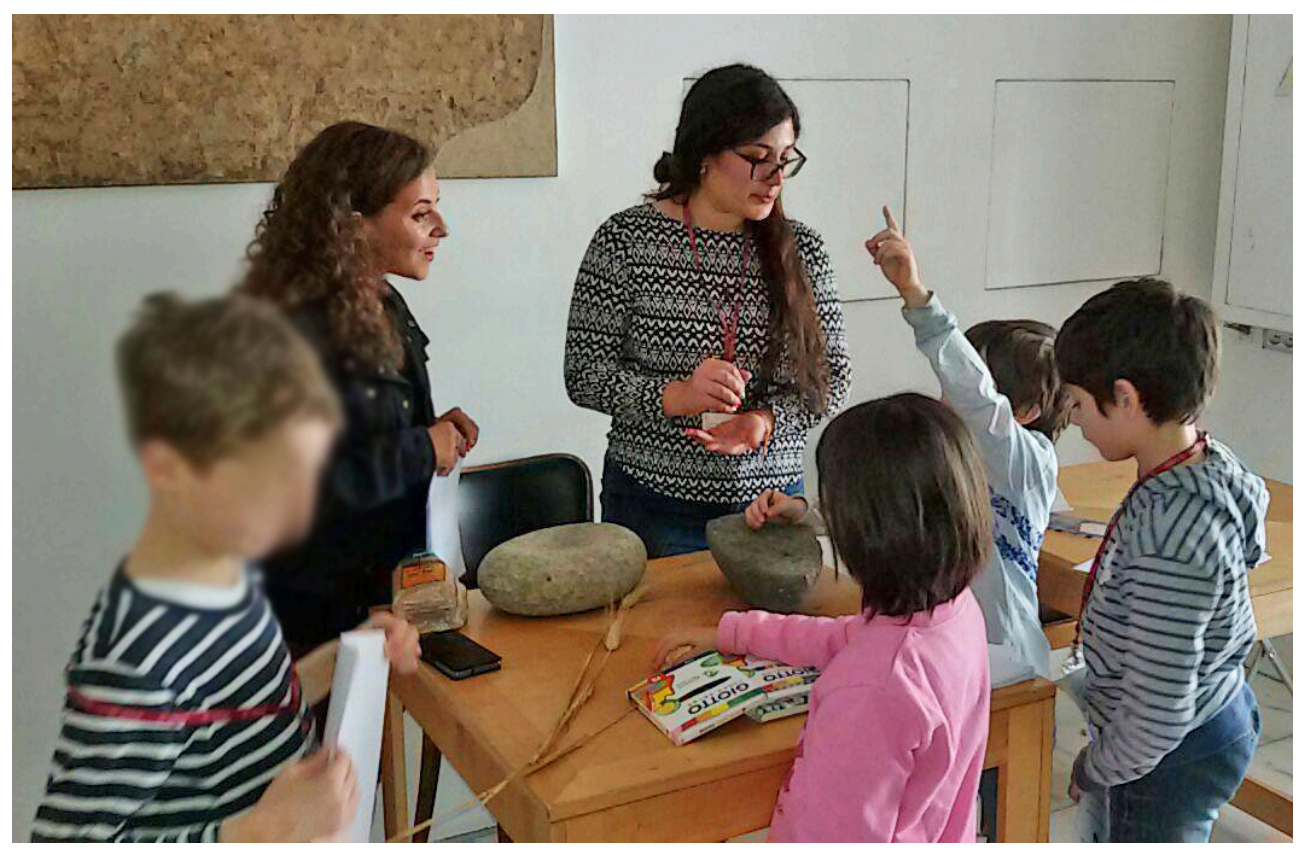

Fig. 2 - Il laboratorio didattico per i ragazzi tra 6 e 12 anni. 
XXI (2017) Bollettino delle attività del Museo del Vicino Oriente, Egitto e Mediterraneo, anno 2017

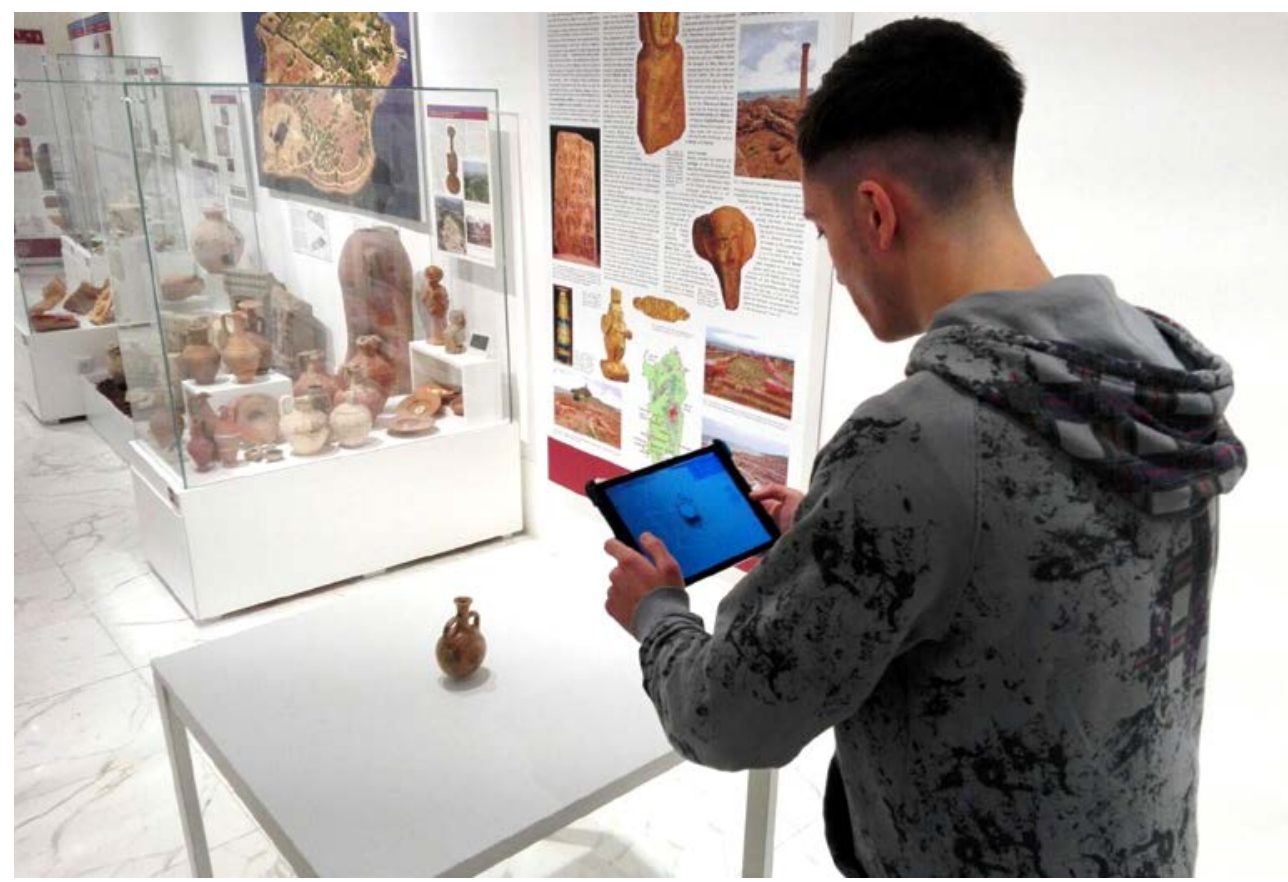

Fig. 3 - Un momento dell'ASL 2017 durante la scansione ottica 3D di uno dei reperti del MVOEM.

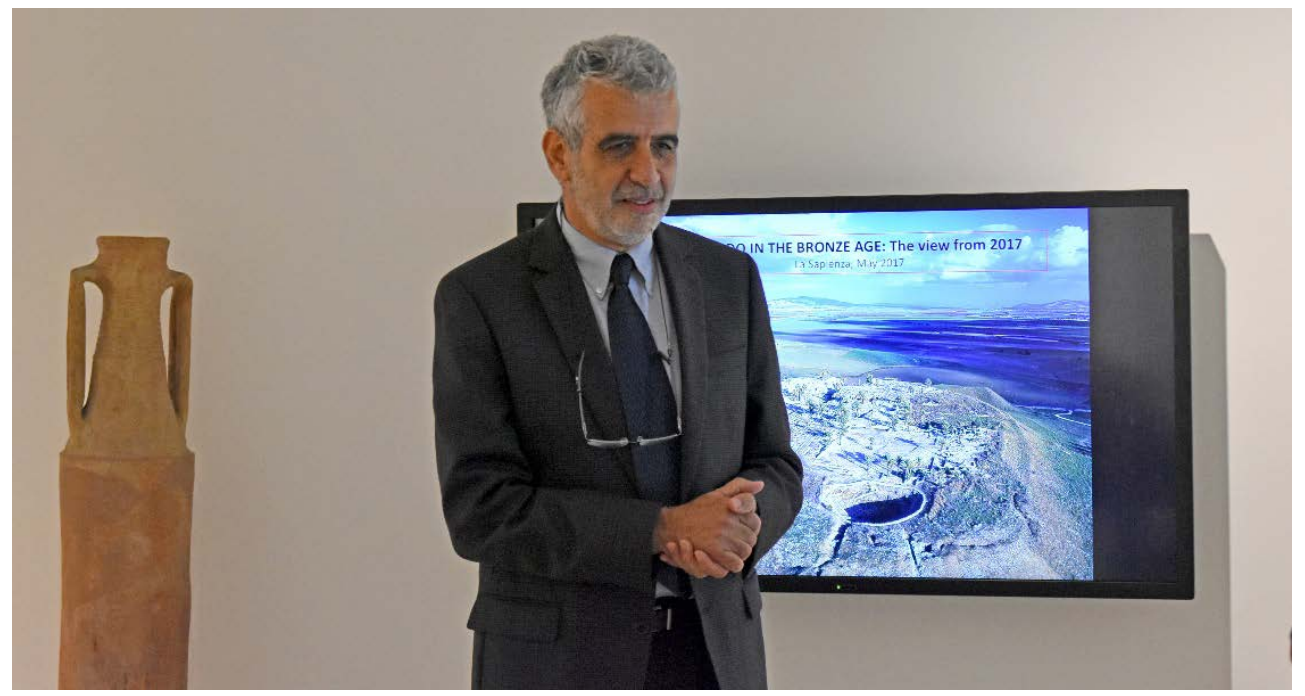

Fig. 4 - Il Prof. Israel Finkelstein durante la Brown Bag Lecture del 10 maggio. 